\title{
Longitudinal Assessment of Global and Regional Left Ventricular Strain in Patients with Multisystem Inflammatory Syndrome in Children (MIS-C)
}

\author{
Michael He ${ }^{1}\left(\mathbb{D} \cdot\right.$ David M. Leone $^{2} \mathbb{D} \cdot$ Richard Frye $^{2} \cdot$ Dina J. Ferdman $^{2} \cdot$ Veronika Shabanova $^{3} \cdot$ Katherine A. Kosiv $^{2}$. \\ Lissa Sugeng ${ }^{4} \cdot$ Erin Faherty $^{2} \cdot$ Ruchika Karnik $^{2}$
}

Received: 30 July 2021 / Accepted: 23 November 2021 / Published online: 7 January 2022

(c) The Author(s), under exclusive licence to Springer Science+Business Media, LLC, part of Springer Nature 2021

\begin{abstract}
Multisystem inflammatory syndrome in children (MIS-C) is one of the most significant sequela of coronavirus disease 2019 (COVID-19) in children. Emerging literature has described myocardial dysfunction in MIS-C patients using traditional and two-dimensional speckle tracking echocardiography in the acute phase. However, data regarding persistence of subclinical myocardial injury after recovery is limited. We aimed to detect these changes with deformation imaging, hypothesizing that left ventricular global longitudinal (GLS) and circumferential strain (GCS) would remain impaired in the chronic phase despite normalization of ventricular function parameters assessed by two-dimensional echocardiography. A retrospective, single-institution review of 22 patients with MIS-C was performed. Fractional shortening, GLS, and GCS, along with regional longitudinal (RLS) and circumferential strain (RCS) were compared across the acute, subacute, and chronic timepoints (presentation, 14-42, and $>42$ days, respectively). Mean GLS improved from $-18.4 \%$ in the acute phase to $-20.1 \%$ in the chronic phase $(p=0.4)$. Mean GCS improved from $-19.4 \%$ in the acute phase to $-23.5 \%$ in the chronic phase $(p=0.03)$. RCS and RLS were impaired in the acute phase and showed a trend towards recovery by the chronic phase, with the exception of the basal anterolateral segment. In our longitudinal study of MIS-C patients, GLS and GCS were lower in the acute phase, corroborating with left ventricular dysfunction by traditional measures. Additionally, as function globally recovers, GLS and GCS also normalize. However, some regional segments continue to have decreased strain values which may be an important subclinical marker for future adverse events.
\end{abstract}

Keywords Coronavirus disease 2019 (COVID-19) · Multisystem inflammatory syndrome in children (MIS-C) · Twodimensional speckle tracking echocardiography $(2 \mathrm{D}-\mathrm{STE}) \cdot$ Longitudinal strain $\cdot$ Circumferential strain

\begin{abstract}
Abbreviations
MIS-C Multisystem inflammatory syndrome in children

COVID-19 Coronavirus disease 2019
\end{abstract}

Michael He

Michael.He@yale.edu

1 Department of Pediatrics, Yale School of Medicine, New Haven, CT, USA

2 Department of Pediatrics, Section of Pediatric Cardiology, Yale School of Medicine, New Haven, CT, USA

3 Department of Pedatrics, Department of Biostatistics, Yale School of Medicine, New Haven, CT, USA

4 Department Medicine, Section of Cardiology, Yale School of Medicine, New Haven, CT, USA
2D-STE Two-dimensional speckle tracking echocardiography

GLS Global longitudinal strain

GCS Global circumferential strain

RLS Regional longitudinal strain

RCS Regional circumferential strain

FS Fractional shortening

KD Kawasaki disease

PICU Pediatric intensive care unit

\section{Introduction}

The pediatric population has been relatively spared from the significant manifesations of acute coronavirus disease of 2019 (COVID-19) when compared to their adult counterparts. However, one significant contrast in this younger 
cohort has been an exaggerated severe inflammatory disease, a newly described syndrome defined as multisystem inflammatory syndrome in children (MIS-C). Though rare, with the most recent United States incidence reported as 2.1 per 100,000 individuals under the age of 21 , MIS-C has led to serious and life threatening complications in otherwise healthy children [1-4].

Although the understanding of the pathophysiology behind MIS-C is still evolving, there have been several clinical cardiac phenotypes [5]. The first described cases had a presentation similar to Kawasaki disease (KD) with coronary artery dilation and occasionally carditis, whereas later cases presented similarly to toxic shock syndrome secondary to an overwhelming immunological response. This included myocarditis, multisystem organ failure, a combination of cardiogenic and/or vasodilatory shock, and varying degrees of coronary artery involvement $[3,5]$. MIS-C does not occur during the acute infection but rather is a delayed post-infectious response, consistently presenting 4-6 weeks after COVID-19 infection or exposure [5, 6]. There has been ample evidence of left ventricular (LV) dysfunction in the acute phase with severe cases presenting as cardiogenic shock, though many patients demonstrate rapid improvement in ventricular function after treatment $[5,6]$. However, to our knowledge, there is limited data on the persistence of subclinical myocardial injury after clinical recovery. We aimed to assess for this using deformation imaging by 2-dimensional speckle tracking echocardiography (2D-STE) at different time points in the clinical course. We hypothesized that LV global longitudinal (GLS) and circumferential strain (GCS) would be impaired in the acute phase and remain diminished in the chronic phase in spite of normalization of ventricular function as assessed by traditional echocardiography.

\section{Methods}

This is a retrospective, single center review of patients who were admitted to the Yale New Haven Children's Hospital. We identified 38 patients between April 2020 and May 2021 with the discharge diagnosis of MIS-C as defined by the United States Centers for Disease Control and Prevention (CDC) criteria [7]. To be included, the patients must have had 2D echocardiographic views of the apical 4-chamber and parasternal short axis at the papillary muscle level suitable for strain analysis at all three time points-acute, sub-acute and chronic phases, defined as at presentation, 14-42 days after, and $>42$ days, respectively. A total of 16 patients were excluded from our study, 10 due to inadequate followup, and 6 who did not have optimal image acquisition to allow for strain analysis. Thus, a total of 22 patients were included in the final analysis. Of the 16 excluded patients, 3 were critically ill requiring vasopressor support, and the remaining 13 did not. Additional subgroup analysis was performed on 8 critically ill patients, defined as admission to the pediatric intensive care unit (PICU) for use of inotropes, vasopressors, or mechanical ventilation. This group is referred to as the PICU group hereon, with the remainder of the 14 patients classified into non-PICU group. Demographic, laboratory, and patient clinical management data was collected through retrospective review of electronic medical record.

During the early pandemic, similar to other institutions, we implemented a handheld echocardiogram protocol using Philips Lumify (Amsterdam, Netherlands) in order to reduce sonographer exposure and allow for easy decontamination [8]. Images obtained included apical 4-chamber and parasternal short axis views in 2-D and M-Mode. Due to the use of Lumify, which allows M-Mode but is unable to measure ejection fraction by Simpson's or 5/6 area-length methods, left ventricular fractional shortening (FS), measured by conventional M-mode, was used for consistency. All Lumify studies were performed by the same experienced sonographer, so the values of FS were thought to have less inter-observer variability. Followup images were obtained using traditional Philips EPIQ CVx. Images obtained were of adequate quality to be evaluated by post-processing strain software. Deformation imaging was performed using vendor-independent TOMTEC software version 1.4.0.64 (Munich, Germany) [9]. GLS from apical 4 chamber view and GCS from LV short axis at the papillary muscle level were compared across time points (Fig. 1). FS was unable to be performed on 4 patients due to missing M-mode evaluation. Though no consensus reference ranges for strain have been established, we defined strain values as abnormal if they were less than $-20 \%$, in keeping with the cutoffs established by others $[10,11]$.

Pilot data was used for sample size power calculations, and timepoints were compared using one-way analysis of variance (ANOVA) with Tukey's multiple comparisons tests. When comparing the PICU versus the non-PICU subgroups, student's t-test was used. Statistical analysis was performed via GraphPad Prism version 9.1.2 (San Diego, CA). For demographic, treatment, and laboratory values, categorical data is presented as frequency (percent), and continuous variables as median (interquartile range [IQR]). Echocardiographic data was assumed to be normally distributed within the population in accordance with other studies, but graphical examination was also performed and demonstrated a normal distribution. As such, echocardiographic data is presented as mean (standard deviation [SD]). Left ventricular wall segments are as defined by American Heart Association 
Longitudinal

Global

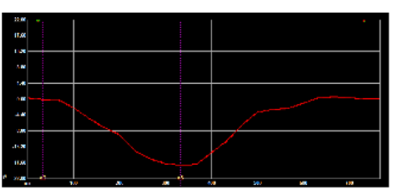

(b)

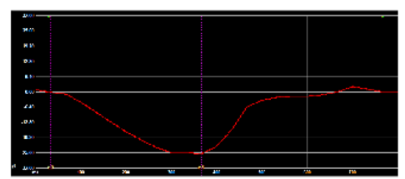

(c)

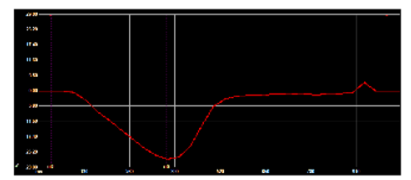

Regional
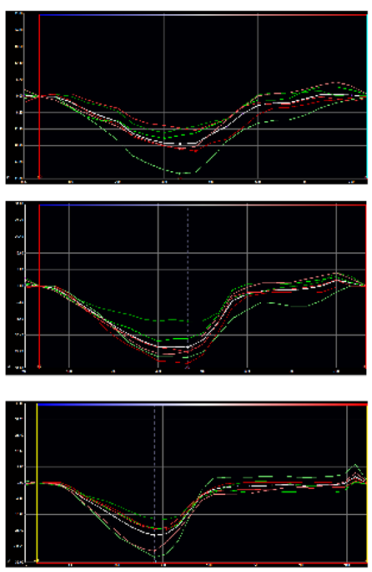

Circumferential
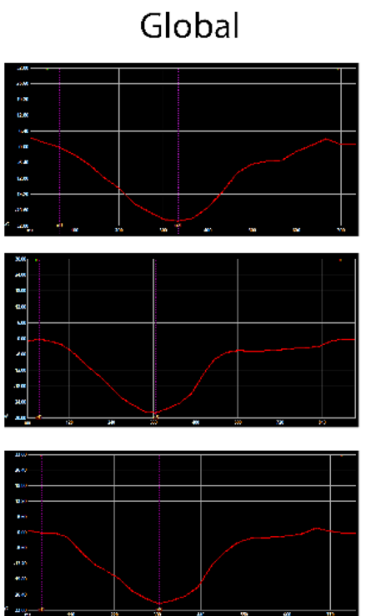

Fig. 1 Longitudinal and circumferential strain tracings in a critically ill patient in the a acute, $\mathbf{b}$ subacute, and $\mathbf{c}$ chronic phase

(AHA) nomenclature [12]. This study was approved by the Yale University institutional review board.

\section{Results}

\section{Demographics and Clinical Presentation}

A total of 22 patients were evaluated. All data is represented as the median (IQR) unless otherwise stated. The cohort age was 7.5 years (5-14) with $13(59 \%)$ being male (Table 1 ). All patients presented with gastrointestinal symptoms and cardiac involvement, as determined by elevation in troponin$\mathrm{T}$ and/or N-terminus pro-brain natriuretic peptide (BNP) levels [7].

The most common treatments in the acute phase included aspirin in 21 (96\%), intravenous immunoglobulin in 18 (82\%), pulsed steroids in 17 (77\%), and anakinra in 7 (32\%). Eight patients (36\%) required PICU admission, with 7 of these requiring vasopressor or inotrope support and 2 requiring mechanical ventilation (Table 1). There was no mortality noted in our study.

Peak laboratory values were most notable for elevated BNP of $5566 \mathrm{pg} / \mathrm{mL}(2083-13,521)$. Inflammatory markers were also elevated with c-reactive protein and ferritin measured at $199 \mathrm{mg} / \mathrm{L} \mathrm{(76-300)} \mathrm{and} 422 \mathrm{ng} / \mathrm{mL}$ (230-602), respectively. In our overall cohort, troponin-T was $0.00 \mathrm{ng} /$ $\mathrm{mL}(0.00-0.13)$, however when stratifying by PICU versus non-PICU cohorts, we noted a significant difference. Within the PICU cohort, peak troponin-T was $0.14 \mathrm{ng} /$ $\mathrm{mL}(0.01-0.26)$, whereas the non-PICU cohort level was $0.00 \mathrm{ng} / \mathrm{mL}(0.00-0.27)[p=0.02]$. Similarly, median BNP within the PICU cohort was $16,739 \mathrm{pg} / \mathrm{mL}(9287-31,491)$
Table 1 Demographic data, clinical presentation, and inpatient interventions

\begin{tabular}{ll}
\hline & $n=22$ \\
\hline Age & $7.5(5-13.5)$ \\
Fale & $13(59 \%)$ \\
& $9(41 \%)$ \\
Organ system involvement & \\
Cardiovascular & \\
Respiratory & $22(100 \%)$ \\
Renal & $7(32 \%)$ \\
Neurologic & $4(18 \%)$ \\
Hematologic & $8(36 \%)$ \\
Gastrointestinal & $13(59 \%)$ \\
Dermatologic & $22(100 \%)$ \\
Pediatric intensive care unit admission & $12(55 \%)$ \\
Mechanical ventilation & \\
Vasopressors & $8(36 \%)$ \\
Milrinone & $2(9 \%)$ \\
Anakinra & $5(23 \%)$ \\
IVIG & $3(14 \%)$ \\
Corticosteroids & $7(32 \%)$ \\
Remdisivir & $18(82 \%)$ \\
Aspirin & $17(77 \%)$ \\
Enoxaparin & $1(4 \%)$ \\
\hline Data is presented as medan (IR) & $22(96 \%)$ \\
& $9(41 \%)$ \\
\hline
\end{tabular}

Data is presented as median (IQR) or $n(\%)$

IVIG intravenous immune globulin

and in the non-PICU group $2736 \mathrm{pg} / \mathrm{mL}$ (1331-5566) $[p=0.002]$. Institutional reference ranges for troponin and BNP were $<0.01 \mathrm{ng} / \mathrm{mL}$ and $<125 \mathrm{pg} / \mathrm{mL}$, respectively. These data are outlined in Table 2. 
Table 2 Peak laboratory values during admission broken down by subgroup

\begin{tabular}{llllr}
\hline & $\begin{array}{l}n=22 \\
\text { All patients }\end{array}$ & $\begin{array}{l}n=16 \\
\text { Non-PICU }\end{array}$ & $\begin{array}{l}n=8 \\
\text { PICU }\end{array}$ & $p$ value \\
\hline Troponin T (ng/mL) & $0.00(0.00-0.13)$ & $0.00(0.00-0.27)$ & $0.14(0.01-0.26)$ & 0.02 \\
BNP $(\mathrm{pg} / \mathrm{mL})^{*}$ & $5566(2083-13,521)$ & $2736(1331-5566)$ & $16,739(9287-31,491)$ & $<0.01$ \\
$\mathrm{CRP}(\mathrm{mg} / \mathrm{L})^{\dagger}$ & $199(76-300)$ & $112(56-300)$ & $256(187-295)$ & 0.09 \\
Ferritin $(\mathrm{ng} / \mathrm{mL})$ & $422(230-602)$ & $294(220-462)$ & $688(545-4769)$ & 0.08 \\
Creatinine $(\mathrm{mg} / \mathrm{dL})$ & $0.49(0.39-0.85)$ & $0.44(0.38-0.54)$ & $1.07(0.54-2.76)$ & 0.06 \\
\hline
\end{tabular}

Data are median (IQR)

$B N P \mathrm{~N}$-terminus pro-brain natriuretic peptide, $C R P \mathrm{C}$-reactive protein

*Maximum value measured for BNP was $70,000 \mathrm{pg} / \mathrm{mL}$

${ }^{\dagger}$ Maximum value measured for CRP was $300 \mathrm{mg} / \mathrm{L}$

\section{D Transthoracic Echocardiography Parameters}

Echocardiographic data including FS and global and regional strain were normally distributed and are presented as mean and standard deviation (Table 3). For our overall study population, mean FS (SD) in the acute, subacute, and chronic phases was 32.2 (7.8), 37.8 (4.4), and 36.0 (6.0) respectively (Table 3). FS showed improvement from the acute to subacute phases $(p=0.03)$ and then remained stable $[p=0.16]$.

Within the PICU cohort, mean FS in the acute, subacute, and chronic phases was 28.7 (8.3), 38.0 (4.0), and 32.5 (5.3), respectively (Table 4). For the non-PICU cohort, FS at the same timepoints were 33.9 (7.3), 37.6 (4.7), and 37.8 (5.7). Though not statistically different, the FS for patients in the PICU subgroup trended lower than those in the non-PICU subgroup during the acute timepoint (Fig. 4).

Coronary artery dilation was seen in $2(9 \%)$ of patients, one of whom had left main and right coronary artery dilation which resolved by the subacute phase. The second had dilation of the right, left main, left anterior descending, and left circumflex arteries during all phases of follow up. No patients demonstrated giant coronary artery aneurysms.
Table 3 LV Strain by 2D-STE in each timepoint for entire cohort

\begin{tabular}{lllllll}
\hline & Acute & Subacute & Chronic & & $p$ value & \\
\cline { 4 - 6 } & & & & $\begin{array}{l}\text { Acute vs. } \\
\text { subacute }\end{array}$ & $\begin{array}{l}\text { Subacute } \\
\text { vs. chronic }\end{array}$ & $\begin{array}{l}\text { Acute vs. } \\
\text { chronic }\end{array}$ \\
\hline Fractional shortening & $32.2(7.8)$ & $37.8(4.4)$ & $36.0(6.0)$ & 0.03 & 0.69 & 0.16 \\
Longitudinal strain & & & & & & \\
Global & $-18.4(4.6)$ & $-21.4(3.4)$ & $-20.1(4.8)$ & 0.06 & 0.57 & 0.40 \\
Basal inferoseptal & $-17.2(5.4)$ & $-21.3(5.3)$ & $-22.3(5.2)$ & 0.03 & 0.79 & 0.01 \\
Mid inferoseptal & $-19.6(6.0)$ & $-23.4(4.3)$ & $-21.6(5.8)$ & 0.05 & 0.50 & 0.44 \\
Apical septal & $-21.1(7.0)$ & $-23.3(7.3)$ & $-20.1(8.2)$ & 0.60 & 0.33 & 0.88 \\
Apical lateral & $-21.4(7.6)$ & $-26.2(5.3)$ & $-24.0(6.5)$ & 0.049 & 0.51 & 0.40 \\
Mid anterolateral & $-18.4(4.9)$ & $-21.6(3.8)$ & $-20.8(4.6)$ & 0.06 & 0.83 & 0.19 \\
Basal anterolateral & $-15.5(5.3)$ & $-17.5(3.9)$ & $-17.0(4.5)$ & 0.31 & 0.93 & 0.51 \\
Circumferential strain & & & & & & \\
Global & $-19.4(6.4)$ & $-23.6(4.5)$ & $-23.5(4.2)$ & 0.02 & 0.99 & 0.03 \\
Mid anterior & $-21.9(10.2)$ & $-26.6(6.4)$ & $-26.7(7.0)$ & 0.14 & 0.99 & 0.14 \\
Mid anterolateral & $-22.1(8.5)$ & $-25.4(8.6)$ & $-24.6(6.7)$ & 0.37 & 0.95 & 0.55 \\
Mid inferolateral & $-19.6(11.1)$ & $-24.3(6.8)$ & $-25.4(6.5)$ & 0.16 & 0.89 & 0.06 \\
Mid inferior & $-19.6(7.5)$ & $-23.9(7.2)$ & $-23.0(7.79)$ & 0.15 & 0.92 & 0.30 \\
Mid inferoseptal & $-18.3(6.8)$ & $-21.6(7.2)$ & $-24.5(6.7)$ & 0.25 & 0.36 & 0.01 \\
Mid anteroseptal & $-22.0(6.7)$ & $-27.9(7.7)$ & $-25.8(6.9)$ & 0.02 & 0.61 & 0.19 \\
\hline
\end{tabular}

Fractional shortening, peak longitudinal and circumferential strain at the acute, subacute, and chronic timepoints. Global and segmental measurements are provided. Data presented as mean (SD). Fractional shortening was not able to be performed on 4/22 subjects due to missing M-mode evaluation 
Table 4 LV Strain by 2D-STE and FS in each timepoint for the PICU and non-PICU subgroups

\begin{tabular}{|c|c|c|c|c|c|c|}
\hline & \multirow[t]{2}{*}{ Acute } & \multirow[t]{2}{*}{ Subacute } & \multirow[t]{2}{*}{ Chronic } & \multicolumn{3}{|l|}{$p$ value } \\
\hline & & & & $\begin{array}{l}\text { Acute vs. } \\
\text { subacute }\end{array}$ & $\begin{array}{l}\text { Subacute } \\
\text { vs. chronic }\end{array}$ & $\begin{array}{l}\text { Acute vs. } \\
\text { chronic }\end{array}$ \\
\hline \multicolumn{7}{|l|}{$P I C U$} \\
\hline Fractional shortening & $28.7(8.3)$ & $38.0(4.0)$ & $32.5(5.3)$ & 0.04 & 0.29 & 0.54 \\
\hline Global longitudinal strain & $-17.2(6.2)$ & $-20.7(4.0)$ & $-17.0(4.0)$ & 0.35 & 0.31 & 0.99 \\
\hline Global circumferential strain & $-18.4(6.1)$ & $-24.5(4.4)$ & $-22.2(5.1)$ & 0.08 & 0.68 & 0.33 \\
\hline \multicolumn{7}{|l|}{ Non-PICU } \\
\hline Fractional shortening & $33.9(7.3)$ & $37.6(4.7)$ & $37.8(5.7)$ & 0.30 & 0.99 & 0.26 \\
\hline Global longitudinal strain & $-19.0(3.5)$ & $-21.8(3.1)$ & $-21.8(4.5)$ & 0.14 & 0.99 & 0.13 \\
\hline Global circumferential strain & $-19.9(6.7)$ & $-23.0(4.6)$ & $-24.1(3.6)$ & 0.25 & 0.84 & 0.09 \\
\hline
\end{tabular}

Fractional shortening, peak longitudinal and circumferential strain in the PICU and non-PICU patients at the acute, subacute, and chronic timepoints. Global and segmental measurements are provided. Data presented as mean (SD). Fractional shortening was not able to be performed on $2 / 8$ subjects in the PICU subgroup and 2/14 in the non-PICU subgroup due to missing M-mode evaluation.

\section{Global Circumferential and Longitudinal Strain}

The mean GLS (SD) values in the acute, subacute, and chronic phase were - 18.4 (4.6), - 21.4 (3.4), and - 20.1 (4.8) respectively. GLS did not meet any statistical change in improvement from the acute to subacute $[p=0.06]$, subacute to chronic [ $p=0.57]$, or acute to chronic [ $p=0.4]$ timepoints, though there was a trend towards improvement (Table 3). The mean GCS (SD) values in acute, subacute, and chronic phase were - 19.4 (6.4), - 23.6 (4.5), and to - 23.5 (4.2), respectively (Fig. 2). GCS showed statistical improvement in both the acute to subacute $[p=0.02]$ and acute to chronic [ $p=0.03]$ timepoints. The mean values for GLS and GCS were decreased in the acute phase but normalized by subacute and chronic phases when compared to previously published age-based normative data [10].

\section{Regional Circumferential and Longitudinal Strain}

In the acute phase, longitudinal strain was depressed in the basal inferoseptal $(-17.2 \pm 5.4)$, mid inferoseptal $(-19.6 \pm 6.0)$, mid anterolateral $(-18.4 \pm 4.9)$, and basal anterolateral segments $(-15.5 \pm 5.3)$. Similarly, circumferential strain at this timepoint was depressed in the mid inferolateral $(-19.6 \pm 11.1)$, mid inferior $(-19.6 \pm 7.5)$, and mid inferoseptal regions ( $-18.3 \pm 6.8$ ) (Fig. 3). All regions except for the basal anterolateral segments showed a trend towards recovery by the subacute and chronic phases (Table 3 ).

\section{Strain Within the PICU Subgroup and Non-PICU Subgroup}

Within the PICU subgroup, GLS was depressed during the acute phase -17.2 (6.2) and showed no improvement to the chronic phase $-17.0(4.0)[p=0.99]$. GCS was similarly depressed in the acute phase at -18.4 (6.1) but showed a trend towards recovery by the chronic phase to -22.2 (5.1) [ $p=0.33]$ (Table 4).

The GLS in the non-PICU group was also depressed in the acute phase at -19.0 (3.5) and trended towards improvement by the chronic phase to -21.8 (4.5), though not statistically significant $[p=0.13]$. GCS in the acute phase was low at -19.9 (6.7) and showed a trend towards normalization by the chronic phase was -24.1 (3.6) [ $p=0.09]$.

When comparing these two subgroups, GLS in the chronic phase was noted to have remained depressed in the PICU subgroup but was improved in the non-PICU subgroup [ $p=0.02]$ (Fig. 4).

\section{Discussion}

\section{Fractional Shortening}

Although not statistically different, patients who required intensive care due to MIS-C had decreased fractional shortening compared to those with milder disease. BNP levels were eight-fold higher on average than those who were not critically ill. There was also a statistically signficant difference in peak troponin-T levels between the two groups, though on average had lower levels than others have presented [6]. This may be due to institutional variations in troponin isoform measured and severity of presentation to our center. However, similar to the work by Matsubara et. al who separated subjects by an elevation in troponin levels, we found similar differences in fractional shortening and ejection fraction [13]. Few patients required mechanical ventilation or vasopressors (9\% and 23\%, 
FS

(a)

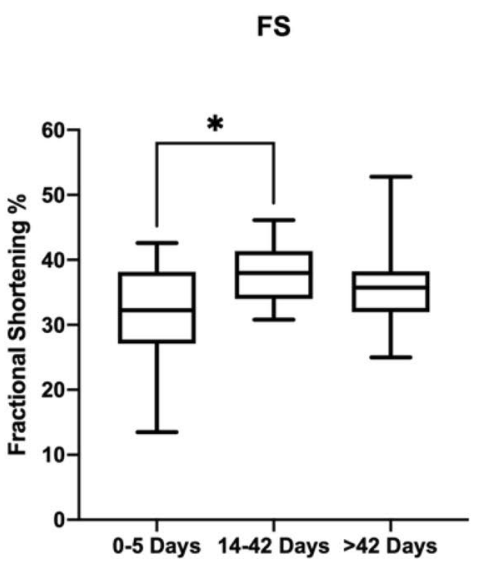

PICU FS

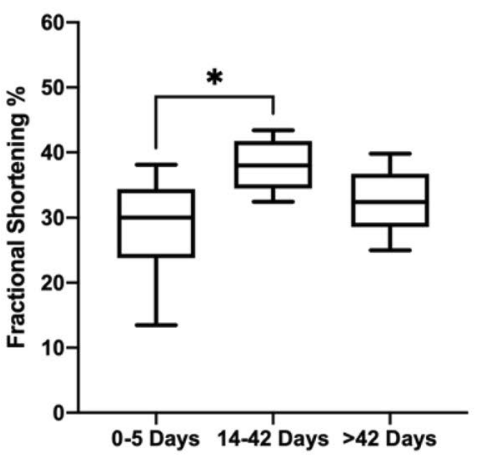

Non-PICU FS

(c)

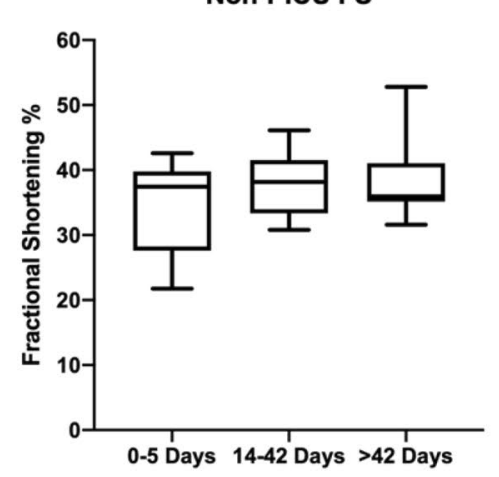

GLS

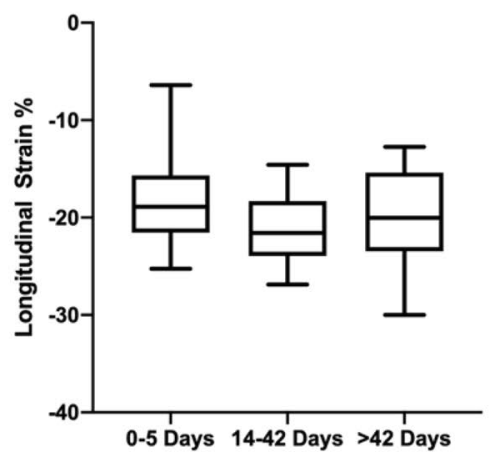

PICU GLS

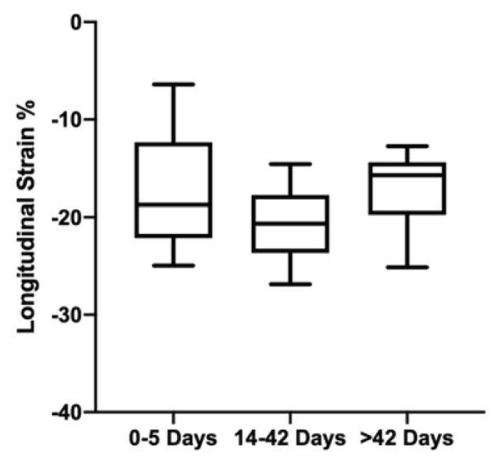

Non-PICU GLS

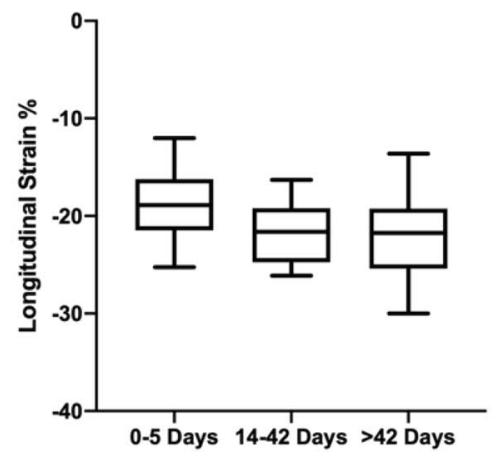

GCS

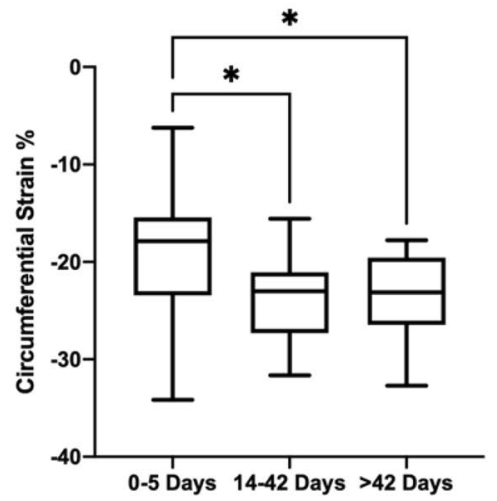

PICU GCS

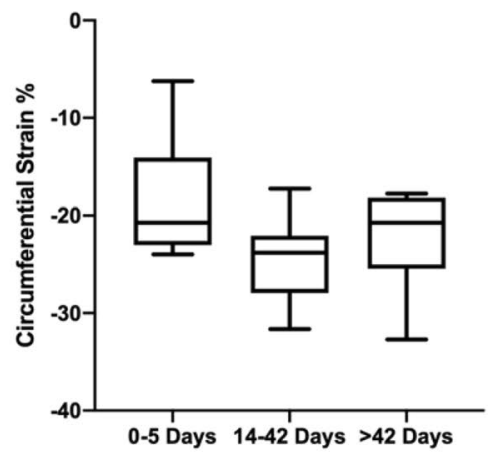

Non-PICU GCS

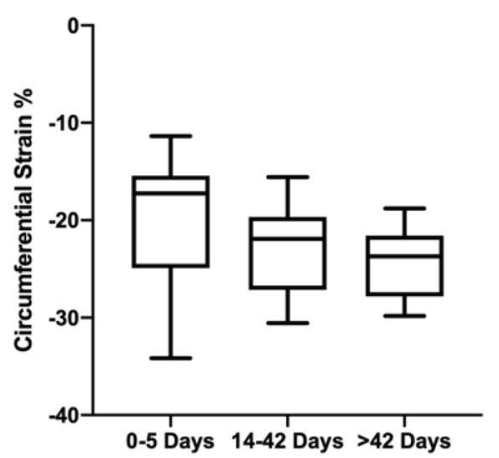

Fig. 2 Changes in LV strain by time point. Fractional shortening (FS), global longitudinal strain (GLS), and global circumferential strain (GVS) in a all patients, b critically ill patients (PICU), and c

respectively). Overall, there was a statistically and clinically significant improvement in fractional shortening across the cohort from the acute to subacute phases.

\section{Global Strain}

In our cohort of MIS-C patients, we found a decrease in both GLS and GCS in the acute phase compared to non-critically ill patients (Non-PICU). *Difference between groups was statistically significant $(p<0.05)$

published normative reference ranges [11]. This impairment is consistent with the clinical presentation of MIS-C that includes evidence of cardiac dysfunction, myocardial injury, elevation of cardiac biomarkers, and occasionally, cardiogenic shock. These findings of abnormal strain have also been described in various other reports [13-16]. Our results showed significant normalization in the GCS from the acute to chronic phase. GLS did not statistically 


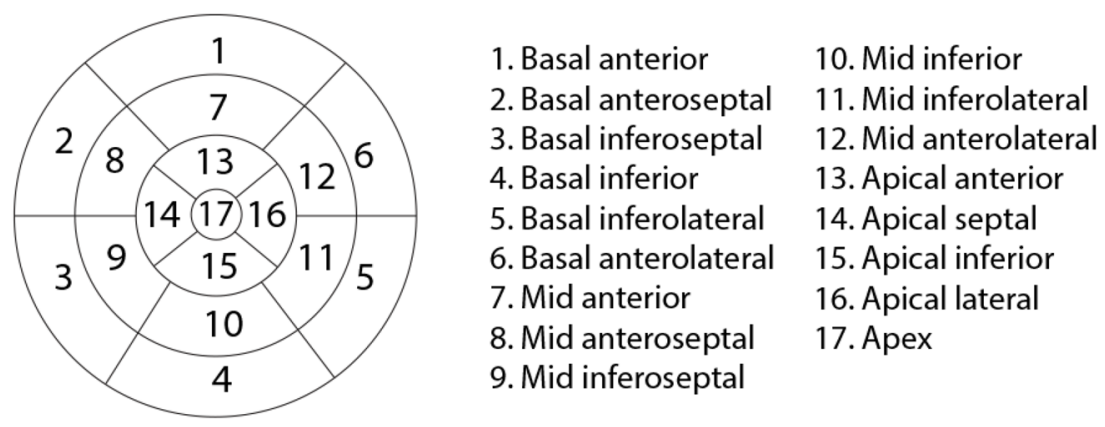

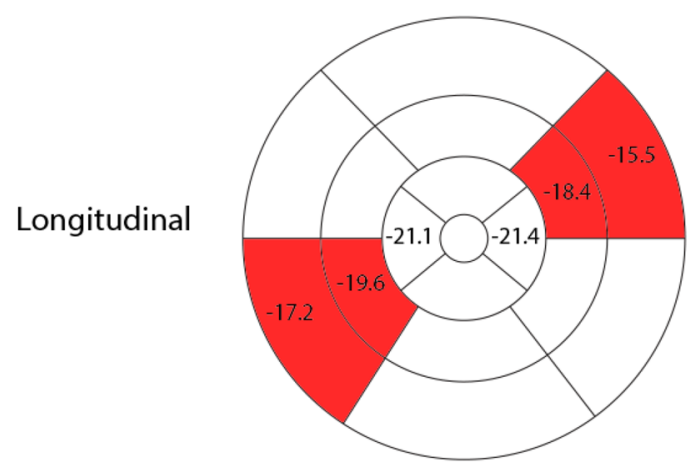

Acute

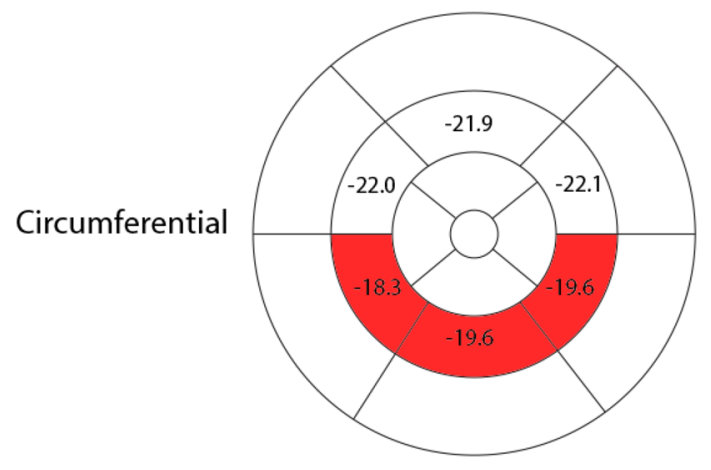

Acute

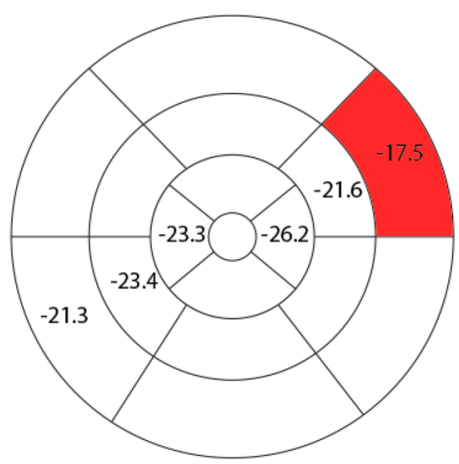

Subacute

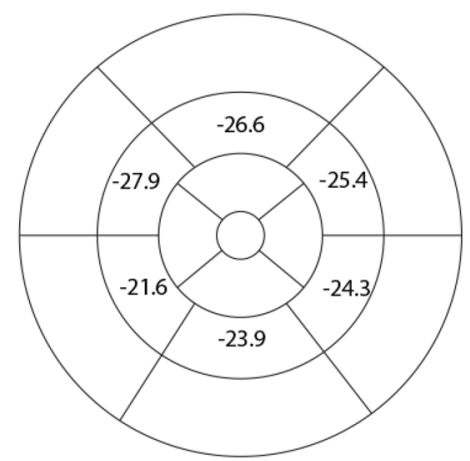

Subacute

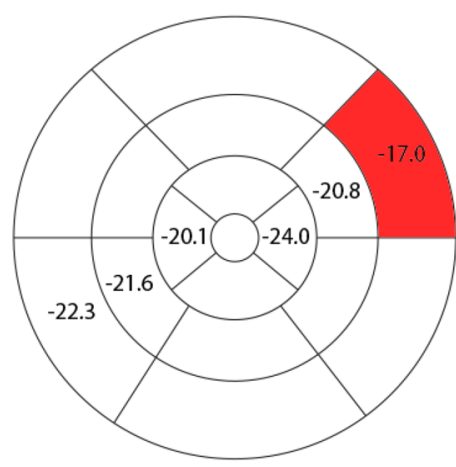

Chronic

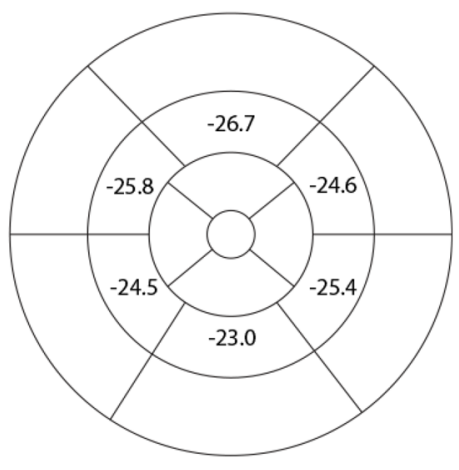

Chronic

Fig. 3 LV regional strain by timepoint. Visualization of the average regional longitudinal strain (RLS) and regional circumferential strain (RCS) in the cohort across the acute, subacute, and chronic phases

change, but on average was considered normal (-20\% or greater) by the chronic phase [10]. This difference in recovery between GCS and GLS was also noted by Gaitonde et. al [14]. Conversely, another study by Theocharis et. al. showed a statistical improvement in GLS from admission to discharge $[14,16]$.

In the PICU subgroup, GLS was more significantly impaired and persisted to be abnormal in the chronic phase despite improvement in the fractional shortening. These results are similar to publications who reported that patients with MIS-C in the lowest tertile for GLS at presentation had persistent depression in GLS at a 10 week follow up [17].

Additionally, GCS in the same subgroup was depressed in the acute phase but trended towards improvement by the chronic phase. On the contrary, those in the non-PICU group, showed lesser degree of impairment at presentation in the GLS and GCS values and had a more robust improvement, which correlated with their overall clinical presenation.

Other publications have also demonstrated impaired GLS and GCS in MIS-C patients [13, 16]. Such findings 
FS Acute Phase

(a)

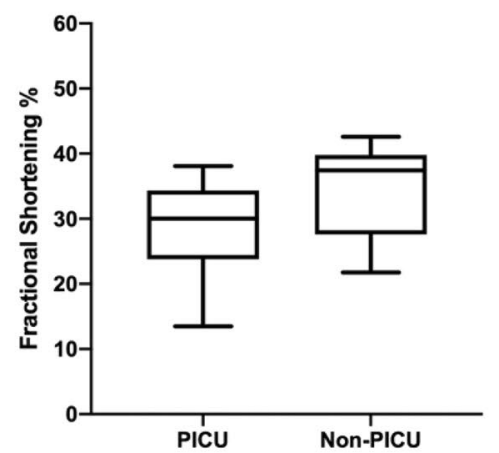

GLS Acute Phase

(b)

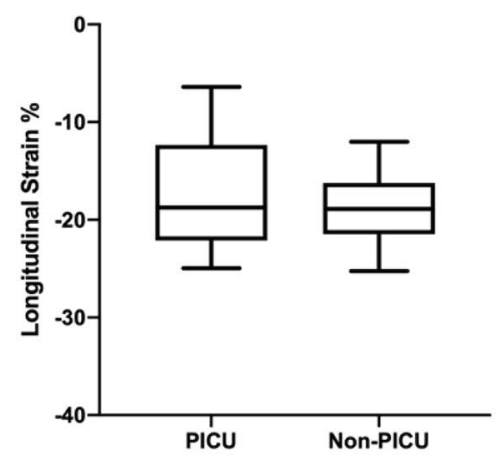

(c)

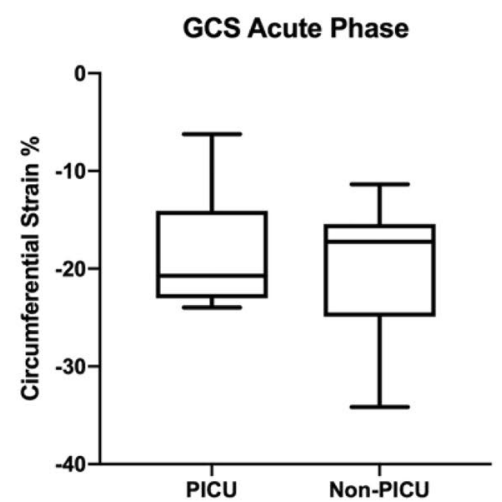

FS Subacute Phase

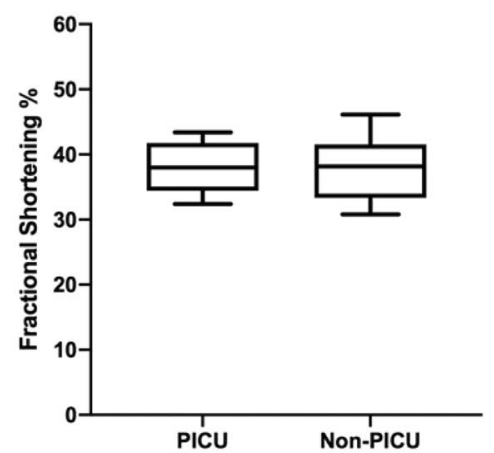

GLS Subacute Phase

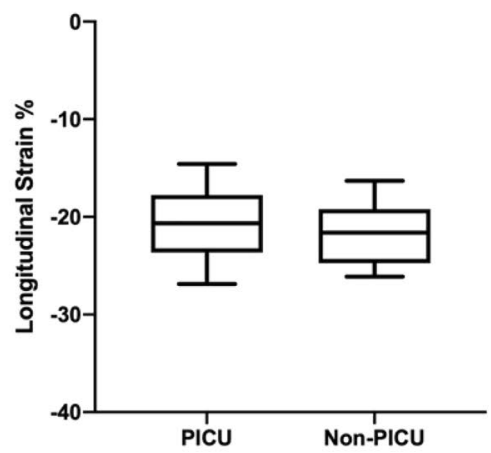

GCS Subacute Phase

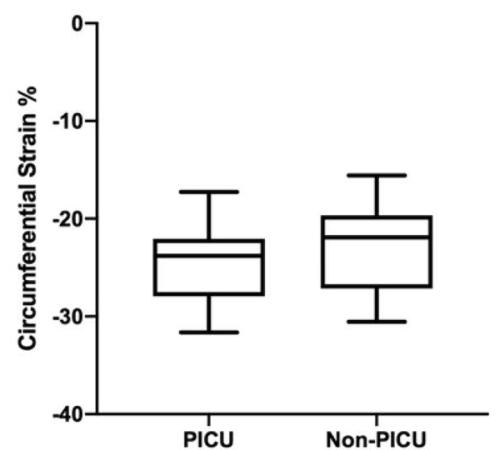

FS Chronic Phase

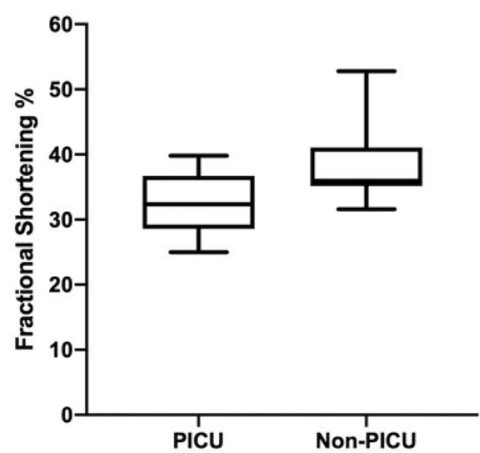

GLS Chronic Phase

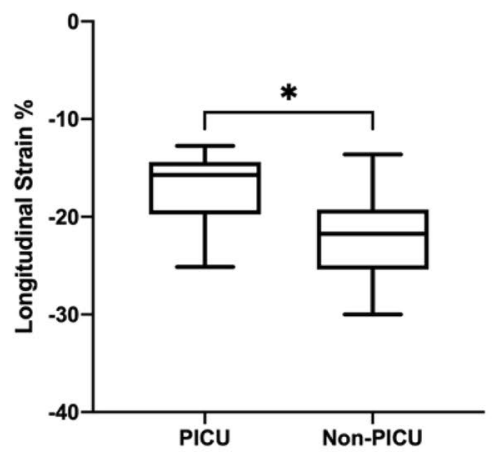

GCS Subacute Phase

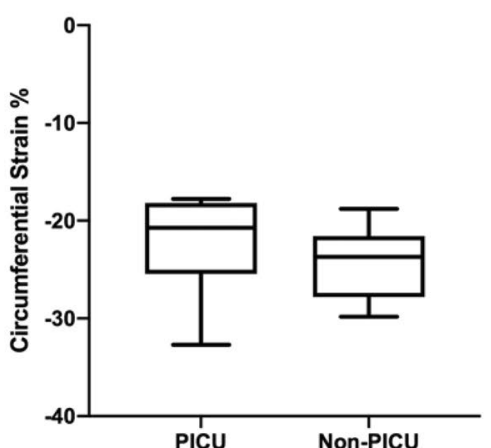

Fig. 4 Changes in LV strain by subgroup. Comparison of a fractional shortening (FS), b global longitudinal strain (GLS), and c global circumferential strain (GCS) between PICU and non-PICU patients within the acute, subacute, and chronic phases. *Difference between groups was statistically significant $(p<0.05)$

\section{Regional Strain}

We noted significant changes in regional LV strain within this cohort. In the acute phase, there was abnormal longitudinal strain, particularly in the anterolateral and inferoseptal regions as outlined in Fig. 3. Evaluation of circumferential strain showed impairment in the three inferior regions (inferoseptal, inferior, and inferolateral). Interestingly, a similar pattern of abnormal regional strain has been reported in of MIS-C. 
both pediatric and adult cases of viral myocarditis which has correlated with regions of myocardial edema and fibrosis on cardiac magnetic resonance imaging [22-24]. This suggests that some of the patterns of myocardial injury with MIS-C may be similar to that of viral myocarditis, though this would require further investigation with cardiac magnetic resonance imaging or endomyocardial biopsy. There are ongoing MRI-based studies for MIS-C patients at this time, which may shed more light on this in the future.

Additionally, this distribution of abnormal regional LV strain roughly correlates to the vascular distributions of the right coronary artery and left circumflex arteries [12]. In comparison, one study of Kawasaki disease patients showed an impairment of strain in the inferolateral and anteroseptal regions, which in turn correlates to the distribution of the left anterior descending artery, which has been the most commonly involved site of aneurysmal change in KD [25, 26]. Though coronary artery changes were overall a rare finding in our cohort, it has been described in patients with MIS-C [6]. Future studies may better elucidate the relationship, if any, between regional wall abnormalities in MIS-C patients and the coronary dilational changes that have been observed.

Finally, the majority of the segments improved to normal values for longitudinal and circumferential strain into the chronic phase. However, the basal anterolateral region had persistently impaired longitudinal strain at $-17 \%$. The clinical significance of this persistent regional impairment in strain is currently not known but does suggest that there may be scar tissue formation and fibrosis that persists despite clinical recovery and normalization of the measured ejection fraction by echocardiography. This may in turn serve as a nidus for development of cardiac arrhythmia, and the identification of this risk, if any, could serve as a future avenue for research.

\section{Limitations}

Our study is limited due to the nature of being a singlecenter retrospective study with a relatively small sample size. This is in part due to the rare occurrence of MIS-C and that even a smaller subset of those have severe disease.

Due to pandemic decontamination constraints and to limit sonographer exposure, at our center we adopted a protocol using the Lumify tablet-based system to capture initial echocardiographic data. The utilization of the Lumify device limited the initial views obtained to apical 4-chamber and parasternal short axis at the level of the papillary muscle, so our reported regional strain analysis may not be representative of all LV segments. When COVID-19 precautions were lifted or the patient was discharged, the subsequent echocardiograms were performed on a traditional machine with comprehensive evaluation. Though ideally a traditional machine would have been used at all timepoints, both the American Society of Echocardiography and the European Association of Cardiovascular Imaging have published statements supporting the use of portable ultrasound to quantify left ventricular function [27, 28]. Additionally, though to our knowledge there have been no head to head comparisons of strain values generated by tablet-based portable devices versus cart-based echocardiographic devices, TOMTEC's vendor-independent calculations have been shown to have minimal differences between devices [9]. By harnessing the power of machine learning and artificial intelligence, software such as TOMTEC may pave the way for more widespread use of tablet-based ultrasound, thereby reducing costs and increasing accuracy, as some have speculated [29]. Finally, within our echocardiography laboratory, we also did an internal quality review of the studies obtained on our tablet during the acute pandemic phase and established that no critical information was compromised [30]. Thus, we feel confident our images were adequate quality for analysis using TOMTEC.

Some of the initial echocardiograms were performed using this protocol and as a result FS data for 4 patients was unavailable and excluded from FS analysis. Similarly, to avoid selection bias, we selected the first echocardiogram obtained for each phase. Some subjects may have had deterioration in their cardiac function after the initial evaluation. Therefore, it is possible the GLS, GCS, and FS values could have been lower in the acute phase. Further, 5 of 8 of the PICU cohort required vasopressors on admission which may have also affected the results of the initial echocardiograms obtained. Finally, a significant proportion of the MIS-C patients seen in our institution were excluded from the study due to lack of adequate imaging (6) or loss to followup (10). The majority (13) of these were not critically ill, whereas the remainder 3 required PICU admission and vasopressor support. We believe these exclusions to be due to chance alone, though there may have been a self-selection bias in which less ill children may be less likely to adhere to regular followup, leading to an overestimation in the long term sequelae of MIS-C.

Evaluation of pediatric left ventricular strain is also limited due to its novelty and lack of guidelines and cutoff measurements for this evaluation. It has also been shown that there is high variability between software manufacturers and normal values have not been established or agreed upon amongst the medical community [10].

\section{Conclusion}

In our longitudinal study of MIS-C patients, we show that global and regional strain are abnormal during acute disease, which correlates with the LV dysfunction as measured 
by FS. This dysfunction was more profound in those with more clinically severe disease. As myocardial and clinical recovery occurs, global and regional strain values also normalize. We also show continued regional abnormalities in the chronic phase which may represent an important marker of subclinical disease and possible increased risk of adverse events in the future. Thus far, there has not been a clear consensus on the optimal frequency and duration of follow-up for these patients with MIS-C. It is unclear what markers are important to determine complete cardiac clinical recovery. Despite clinical improvement in symptoms and fractional shortening, we do not fully understand the implications of anormal regional strain that may represent subclinical myocardial injury. For now, our data seems to emphasize the need for continued long-term follow-up of these patients.

Author Contributions All authors contributed to the study conception and design. Material preparation, data collection, and statistical analysis were performed by MH. The first draft of the manuscript was written by MH with major revisions of the draft and tables by DL. RF also contributed to data collection and VS aided in statistical design and analysis. DL, RK, DF, and EF contributed to clinical aspects of the study design and interpretation. All authors commented on previous versions of the manuscript and approved the final manuscript.

\section{Funding None.}

Availability of Data and Material The data that support the findings of this study are available from the corresponding author, $\mathrm{MH}$, upon reasonable request.

\section{Declarations}

\section{Conflict of interest None.}

Ethical Approval Yale Institutional Review Board Approval Number 2000029338.

\section{References}

1. Riphagen S, Gomez X, Gonzalez-Martinez C et al (2020) Hyperinflammatory shock in children during COVID-19 pandemic. Lancet 395:1607-1608. https://doi.org/10.1016/S0140-6736(20) 31094-1

2. Verdoni L, Mazza A, Gervasoni A et al (2020) An outbreak of severe Kawasaki-like disease at the Italian epicentre of the SARSCoV-2 epidemic: an observational cohort study. Lancet 395:17711778. https://doi.org/10.1016/S0140-6736(20)31103-X

3. Feldstein LR, Rose EB, Horwitz SM et al (2020) Multisystem inflammatory syndrome in U.S. children and adolescents. N Engl J Med 383:334-346. https://doi.org/10.1056/NEJMoa2021680

4. Belay ED, Abrams J, Oster ME et al (2021) Trends in geographic and temporal distribution of US children with multisystem inflammatory syndrome during the COVID-19 pandemic. JAMA Pediatr 175:837-845. https://doi.org/10.1001/jamapediatrics.2021.0630
5. Friedman KG, Harrild DM, Newburger JW (2020) Cardiac dysfunction in multisystem inflammatory syndrome in children: a call to action. J Am Coll Cardiol 76:1962-1964. https://doi.org/ 10.1016/j.jacc.2020.09.002

6. Alsaied T, Tremoulet AH, Burns JC et al (2021) Review of cardiac involvement in multisystem inflammatory syndrome in children. Circulation 143:78-88. https://doi.org/10.1161/CIRCULATIO NAHA.120.049836

7. (2020) Multisystem Inflammatory Syndrome in Children (MISC) associated with Coronavirus Disease 2019 (COVID-19). In: Centers Dis. Control Prev. Heal. Alert Netw. https://emergency. cdc.gov/han/2020/han00432.asp

8. McMahon SR, De Francis G, Schwartz S et al (2020) Tabletbased limited echocardiography to reduce sonographer scan and decontamination time during the COVID-19 pandemic. J Am Soc Echocardiogr 33:895-899. https://doi.org/10.1016/j.echo. 2020.05.005

9. Nagata Y, Takeuchi M, Mizukoshi K et al (2015) Intervendor variability of two-dimensional strain using vendor-specific and vendor-independent software. J Am Soc Echocardiogr Off Publ Am Soc Echocardiogr 28:630-641. https://doi.org/10.1016/j. echo.2015.01.021

10. Levy PT, Machefsky A, Sanchez AA et al (2016) Reference ranges of left ventricular strain measures by two-dimensional speckle-tracking echocardiography in children: a systematic review and meta-analysis. J Am Soc Echocardiogr. https://doi. org/10.1016/j.echo.2015.11.016

11. Jashari H, Rydberg A, Ibrahimi P et al (2015) Normal ranges of left ventricular strain in children: a meta-analysis. Cardiovasc Ultrasound 13:37. https://doi.org/10.1186/s12947-015-0029-0

12. Cerqueira MD, Weissman NJ, Dilsizian V et al (2002) Standardized myocardial segmentation and nomenclature for tomographic imaging of the heart. Circulation 105:539-542. https:// doi.org/10.1161/hc0402.102975

13. Matsubara D, Kauffman HL, Wang Y et al (2020) Echocardiographic findings in pediatric multisystem inflammatory syndrome associated With COVID-19 in the United States. J Am Coll Cardiol 76:1947-1961. https://doi.org/10.1016/j.jacc.2020. 08.056

14. Gaitonde M, Ziebell D, Kelleman MS et al (2020) COVID-19-related multisystem inflammatory syndrome in children affects left ventricular function and global strain compared with Kawasaki disease. J Am Soc Echocardiogr Off Publ Am Soc Echocardiogr 33:1285-1287. https://doi.org/10.1016/j.echo.2020.07.019

15. Kobayashi R, Dionne A, Ferraro A et al (2021) Detailed assessment of left ventricular function in multisystem inflammatory syndrome in children using strain analysis. CJC Open. https:// doi.org/10.1016/j.cjco.2021.02.012

16. Theocharis P, Wong J, Pushparajah K et al (2020) Multimodality cardiac evaluation in children and young adults with multisystem inflammation associated with COVID-19. Eur Heart J Cardiovasc Imaging. https://doi.org/10.1093/ehjci/jeaa212

17. Sanil Y, Misra A, Safa R et al (2021) Echocardiographic indicators associated with adverse clinical course and cardiac sequelae in multisystem inflammatory syndrome in children with coronavirus disease 2019. J Am Soc Echocardiogr Off Publ Am Soc Echocardiogr. https://doi.org/10.1016/j.echo.2021.04.018

18. Nestaas E, Støylen A, Brunvand L, Fugelseth D (2011) Longitudinal strain and strain rate by tissue Doppler are more sensitive indices than fractional shortening for assessing the reduced myocardial function in asphyxiated neonates. Cardiol Young 21:1-7. https://doi.org/10.1017/S1047951109991314

19. Streeter DD, Spotnitz HM, Patel DP et al (1969) Fiber orientation in the canine left ventricle during diastole and systole. Circ Res 24:339-347. https://doi.org/10.1161/01.RES.24.3.339 
20. Halliday BP, Senior R, Pennell DJ (2021) Assessing left ventricular systolic function: from ejection fraction to strain analysis. Eur Heart J 42:789-797. https://doi.org/10.1093/eurheartj/ehaa587

21. Stokke T, Hasselberg N, Smedsrud M et al (2017) Geometry as a confounder when assessing ventricular systolic function. J Am Coll Cardiol 70:942-954. https://doi.org/10.1016/j.jacc.2017.06. 046

22. Chinali M, Franceschini A, Ciancarella P et al (2020) Echocardiographic two-dimensional speckle tracking identifies acute regional myocardial edema and sub-acute fibrosis in pediatric focal myocarditis with normal ejection fraction: comparison with cardiac magnetic resonance. Sci Rep 10:11321. https://doi.org/10.1038/ s41598-020-68048-5

23. L $ø$ gstrup BB, Nielsen JM, Kim WY, Poulsen SH (2016) Myocardial oedema in acute myocarditis detected by echocardiographic 2D myocardial deformation analysis. Eur Hear J Cardiovasc Imaging 17:1018-1026. https://doi.org/10.1093/ehjci/jev302

24. Meindl C, Paulus M, Poschenrieder F et al (2021) Patients with acute myocarditis and preserved systolic left ventricular function: comparison of global and regional longitudinal strain imaging by echocardiography with quantification of late gadolinium enhancement by CMR. Clin Res Cardiol. https://doi.org/10.1007/ s00392-021-01885-0

25. Lin Z, Zheng J, Chen W et al (2020) Assessing left ventricular systolic function in children with a history of Kawasaki disease. BMC Cardiovasc Disord 20:131. https://doi.org/10.1186/ s12872-020-01409-0
26. Onouchi Z, Shimazu S, Kiyosawa N et al (1982) Aneurysms of the coronary arteries in Kawasaki disease. An angiographic study of 30 cases. Circulation 66:6-13. https://doi.org/10.1161/01.CIR. 66.1 .6

27. Cardim N, Dalen H, Voigt J-U et al (2019) The use of handheld ultrasound devices: a position statement of the European Association of Cardiovascular Imaging (2018 update). Eur Hear J Cardiovasc Imaging 20:245-252. https://doi.org/10.1093/ehjci/jey145

28. Spencer KT, Kimura BJ, Korcarz CE et al (2013) Focused cardiac ultrasound: recommendations from the American Society of Echocardiography. J Am Soc Echocardiogr Off Publ Am Soc Echocardiogr 26:567-581. https://doi.org/10.1016/j.echo.2013. 04.001

29. Akkus Z, Aly YH, Attia IZ et al (2021) Artificial intelligence (AI)-empowered echocardiography interpretation: a state-of-theart review. J Clin Med. https://doi.org/10.3390/jcm10071391

30. Leone DM, Ghiroli S, Karnik R et al (2021) Abstract 11298: tablet-based echocardiography is a valuable tool to effectively evaluate patients with Sars-Cov-2 while limiting sonographer exposure. Circulation 144:A11298-A11298. https://doi.org/10. 1161/circ.144.suppl_1.11298

Publisher's Note Springer Nature remains neutral with regard to jurisdictional claims in published maps and institutional affiliations. 